\title{
Spin-Torque-Nano-Oscillator based neuromorphic computing assisted by laser
}

\author{
Hooman Farkhani*, Tim Böhnert ${ }^{\dagger}$, Mohammad Tarequzzaman ${ }^{\dagger}$, Diogo Costa $^{\dagger}$, Alex Jenkins ${ }^{\dagger}$, Ricardo Ferreira ${ }^{\dagger}$ and Farshad \\ Moradi* \\ Emails: farkhani@eng.au.dk, tim.boehnert@inl.int, tarequzzaman@gmail.com, zediogo.costa@gmail.com, \\ alex.jenkins@inl.int, Ricardo.ferreira@inl.int,moradi@eng.au.dk \\ *Integrated Circuits and Electronics Laboratory, Department of Engineering, Aarhus University, Aarhus, Denmark \\ †International Iberian Nanotechnology Laboratory, INL, Braga, Portugal
}

\begin{abstract}
In spintronic-based neuromorphic computing systems (NCSs), the switching of magnetic moment in a magnetic tunnel junction (MTJ) or magnetic oscillation in spin torque nano-oscillator (STNO) is used to mimic biological neuron firing. Although using STNO reduces the power consumption significantly, still there is a huge gap between the power consumption of spintronic-based NCS and brain due to the high bias current. In this paper, the power consumption of the proposed STNO-based NCS is reduced by thermally assisting the STNO oscillation through a microwatt nanosecond laser pulse. The experimental results show the power consumption of STNOs in NCS reduces by $56 \%$ by heating them up to $100^{\circ} \mathrm{C}$. The total power consumption of the proposed laser assisted oscillation-based NCS (LAO-NCS) is reduced by $46 \%$ at $100^{\circ} \mathrm{C}$ compared with a typical STNO-based NCS at room temperature.
\end{abstract}

Keywords- neuromorphic computing system, laser, power efficient, STNO

\section{INTRODUCTION}

The grand challenge of exascale computing, $10^{18}$ operations/second, calls for a dramatic change in hardware of the current petascale supercomputers. A paradigm shift is required to tackle the issue of processing the huge amount of images and videos that is the most time and power consuming task for the existing Von-Neumann computing machines (VNC). The most promising solution is the brain-inspired systems, so-called neuromorphic computing systems (NCSs), which overcomes the limitation of the word-at-a-time thinking of the VNCs by massive parallel data processing similar to the brain [1-5]. An NCS includes many parallel processors (neurons) communicating using simple messages (spikes) through programmable memory units (synapses). The current implementation of NCSs using CMOS technology is area and power inefficient [6]. Such inefficiencies have driven a significant effort to investigate beyond-CMOS NCSs. The spin-based devices integrated with electronics (i.e. spintronics) have opened a door for designers to implement low-power high-density NCSs. In spintronic-based NCSs, magnetic switching in magnetic tunnel junction (MTJ) [6] or magnetic oscillation in spin torque nano-oscillator (STNO) [7][8] is used to mimic neuron firing. While using oscillation of magnetic moment decreases the power consumption by an order of magnitude compared with magnetic moment switching (critical current: $\sim 10^{6} \mathrm{Acm}^{-2}[9]$ vs $\sim 10^{-7} \mathrm{Acm}^{-2}[10]$ ), still there is a huge gap between spintronic-based NCS and brain in terms of power consumption and speed. This is due to high power consumption and low speed of the traditional way of oscillating the magnetic moment through bias current. Hence, there is a crucial need for eliminating or decreasing the bias current in spintronic-based NCSs.

This work was supported by the Marie Sklodowska-Curie Individual Fellowship (IF) under contract number 751089.

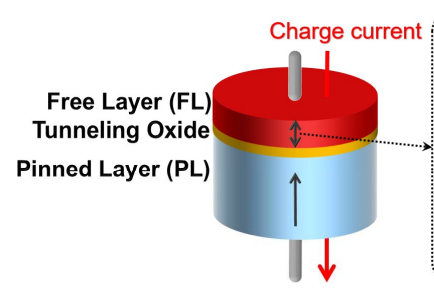

(a)

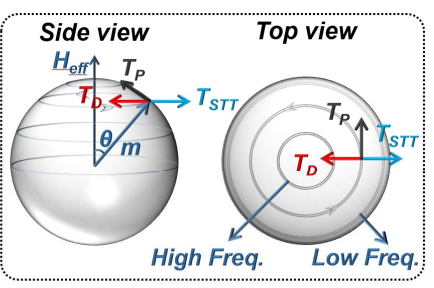

(b)
Fig. 1. (a) The schematic view of a MTJ as STNO and (b) the magnetization direction of MTJ free layer and torques acting on it.

In this paper, benefiting from heating up the STNO with a microwatt-nanosecond laser pulse, for the first time, we propose to design a laser-assisted oscillation-based NCS (LAO-NCS) to improve power consumption of the state-ofthe-art NCSs by at least $46 \%$; filling the huge gap of power consumption between the Brain and the NCSs.

\section{A. Spin Torque Nano Oscillator}

The schematic of the STNO is shown in Fig. 1 (a). It consists of a Pinned Layer (PL) with fixed magnetization and a Free Layer (FL) with changeable magnetization direction, which are separated by a tunneling oxide layer such as $\mathrm{MgO}$, $\mathrm{Al}_{2} \mathrm{O}_{3}$ etc. Fig. 1 (b) shows the magnetization direction of the free layer $(\mathrm{m})$ and different torques acting on it [7]. $\mathrm{T}_{\mathrm{P}}$ describes the precession torque that leads to oscillation of $\mathrm{m}$. $T_{D}$ is the damping torque that aligns $m$ with $H_{\text {eff }}$ and $T_{\text {STT }}$ is the spin-transfer torque caused by a bias current [8]. The interaction of $\mathrm{T}_{\mathrm{STT}}$ and $\mathrm{T}_{\mathrm{D}}$ determines the oscillatory orbit of $\mathrm{m}$. As $\mathrm{T}_{\mathrm{STT}}$ increases, $\mathrm{m}$ will be placed in an orbit farther than $\mathrm{H}_{\text {eff, which will lead to a lower frequency oscillation of } \mathrm{m} \text { as }}$ shown in Fig. 1 (b) [11]. This is shown experimentally and through simulation that the frequency of the STNO can be locked to the frequency of an RF current passing through it [12][13] or an external oscillating RF field [14]. Moreover, the frequency of two STNOs can lock if they are close to each other [15]. In STNO-based NCS, frequency locking of STNO and comparing its output power with a threshold power are two mechanisms used to implement neuron firing. However, in all cases, an extremely high DC current (bias current) has to flow through the STNO to generate the required $\mathrm{T}_{\mathrm{STT}}$.

\section{B. Effect of Raising Temperature on STNO}

The dynamic behavior of the FL magnetic moment is modeled using Landau-Lifshitz-Gilbert-Slonczewski (LLGS) equation as follows [16]:

$$
\frac{\left(1+\alpha^{2}\right)}{|\gamma|} \frac{\partial \widehat{m}}{\partial t}=-\widehat{m} \times \vec{H}_{E F F}-\alpha \widehat{m} \times \widehat{m} \times \vec{H}_{E F F}
$$

where $\gamma, \alpha$ and $\widehat{m}$ are the gyromagnetic ratio, Gilbert damping factor and magnetization of FL, respectively. $\vec{H}_{E F F}$ is the effective magnetic field acting on FL described by 
$\vec{H}_{E F F}=\vec{H}_{U A}+\vec{H}_{e x}+\vec{H}_{S T T}+\vec{H}_{T F}$, where $\vec{H}_{U A}, \vec{H}_{e x}, \vec{H}_{S T T}$ and $\vec{H}_{T F}$ are uniaxial anistropy field, external magnetic field, current induced spin-transfer torque field and thermal fluctuations field, respectively. Increasing the temperature affects the dynamic behavior of the FL through decreasing the magnetization saturation $\left(\mathrm{M}_{\mathrm{S}}\right)$ of it, decreasing the resistance of the STNO and increasing the dispersion of the initial deviation of the magnetic moment from easy axis due to higher thermal fluctuations.

Magnetization saturation: It is shown theoretically [17] and experimentally [18] that the dependency of $\mathrm{M}_{\mathrm{S}}$ can be well described by Bloch's law as follows:

$$
M_{S}(T)=M_{S}(0)\left(1-\left(T / T^{*}\right)^{\frac{3}{2}}\right)
$$

where $T$ is the absolute temperature in Kelvin and $M_{S}(0)$ is the saturation magnetization at $0 \mathrm{~K}$, and $T^{*}$ is a fitting factor. Equation (2) shows that increasing the temperature decreases $\mathrm{M}_{\mathrm{S}}(\mathrm{T})$. This leads to a degradation of the uniaxial anisotropy field, which decreases the minimum current required for FL magnetic oscillation.

Resistance: Two tunneling mechanisms contribute to the STNO resistance including electron spin-polarized direct elastic tunneling and spin independent tunneling. The total conductance of the STNO can be described as [19]

$$
G(\theta)=G_{T}\left[1+P_{1} P_{2} \cos \theta\right]+G_{S I}
$$

where $\theta$ is the angle between the magnetization of the FL and the PL. $\mathrm{P}_{1}$ and $\mathrm{P}_{2}$ are the effective tunneling spin polarization of the magnetic layers. $G_{T}$ is the pre-factor for direct elastic tunneling. The $\mathrm{P}_{1}, \mathrm{P}_{2}$ and $\mathrm{G}_{\mathrm{T}}$ parameters are temperaturedependent. Elevating the temperature increases $\mathrm{G}_{\mathrm{T}}$ and reduces $P_{1}$ and $P_{2}$ [19]. As a result, $R_{P}$ is almost independent of temperature while $\mathrm{R}_{\mathrm{AP}}$ reduces approximately linearly with temperature. This has been experimentally shown in [19-21].

Thermal fluctuations: The effect of temperature on random fluctuating field can be modeled by $\vec{H}_{T F}$ while its x, y and $\mathrm{z}$ components have uncorrelated Gaussian distribution with zero mean and $\sqrt{\left(2 \alpha k_{B} T\right) /\left(\gamma M_{S} V \Delta t\right)}$ standard deviation [22-24]. $\alpha, \mathrm{k}_{\mathrm{B}}, \gamma, \mathrm{V}$ and $\Delta \mathrm{t}$ are Gilbert damping parameter, Boltzmann's constant, gyromagnetic ratio, the volume of the FL and the integration time step. Elevating the temperature increases the dispersion of $\vec{H}_{T F}$, which leads to an easier oscillation of FL magnetic moment. In order to explore the mentioned effects on oscillation behavior of STNO at elevated temperatures, different characteristics of STNO (resistance, TMR and output power of the oscillation) have been measured at different temperatures from $27^{\circ} \mathrm{C}$ up to $100^{\circ} \mathrm{C}$ in section III.

\section{Memristor Behavior at Elevated Temperature}

The conduction mechanism of the memristor can be modeled by two parallel conduction mechanisms including hopping conduction and Schottky thermionic emission [25] as follows:

$$
I_{\text {total }}=\underbrace{2 e l v_{p h} N k_{B} T e^{\left(-\frac{W}{k_{B} T}\right)} e^{\left(-\frac{2 l}{\zeta}\right)} \sinh \left(\frac{q l F}{k_{B} T}\right)}_{I_{\text {hop }}}+\underbrace{A T^{2} e^{\left(-\frac{\phi_{B 0}-\beta \sqrt{F}}{k_{B} T}\right)}}_{I_{S c h}}
$$

where, $k_{B}$ is the Boltzmann constant, $l$ is the hopping distance, $W$ is the hopping energy, $\zeta$ is the wave function localization, $F$ is the applied field (converts from $\mathrm{V}$ ), $T$ is the temperature, $v_{p h}$ is the vibrational phonon frequency, $A$ is the reduced effective Richardson constant multiplied by active device area, $\phi_{B 0}$ is the barrier height, and $\beta$ is the barrier lowering factor. $N$ is proportional to the density of electrons in the conduction path multiplied by the relevant conducting area. Based on this model, which is well-fitted with experimental results, the temperature dependence of memristor resistance can be divided into two regions called cold and hot regions [25]. In the cold region $(T \leqslant 350 \mathrm{~K})$, the state-dependent hopping conduction is dominant and the resistance of memristor is almost temperature insensitive. In the hot region, however, the Schottky emission of electrons determines the hot current and the memristor's resistance rapidly decreases with raising the temperature [25]. Noted, the trend of cold region (temperature independent) and hot region (temperature dependent) is independent of initial resistance state of memristor [25]. However, the resistance change of memristor in hot region depends on memristor's initial resistance.

\section{PROPOSED LASER ASSISTED NEUROMORPHIC COMPUTING SYSTEM}

Our novel envisioned LAO-NCS is shown in Fig. 2, which is a crossbar array of programmable memristors as synapses and the STNOs, assisted thermally by a narrow laser-pulse, as neurons. The resistance of the memristors can be tuned using an electric signal passing through them. The NCS operation starts with a calibration phase in which the NCS temperature will be elevated to $100^{\circ} \mathrm{C}$ and stabilized. Then, the NCS is ready for operation and the processing phase will be started. The processing phase can be divided into two steps including stimulation and recovery which will be repeated in sequence. In the stimulation step, the crossbar array sums weighted input currents passing them to the STNOs, which are already set in AP-state. In case, the weighted input currents are sufficiently large, the FL magnetic moment of STNO starts to oscillate that will be detected by a sensing circuit immediately [26-28], and translated to neuron's firing. Immediately after detecting the STNO oscillation, the recovery step begins. In the recovery step, the input corresponding to the fired neuron will be activated in the post NCS. Noted, one of the advantages of using oscillation instead of magnetic moment switching is that there is no need for switching back the FL magnetization. Hence, the recovery step can be done in a very short time ( $\sim 600 \mathrm{ps})$ compared with magnetic moment switching ( 2ns) without extra energy consumption for switching back the magnetic moment. In our approach, the energy consumption needed for starting the STNO oscillation will be lowered

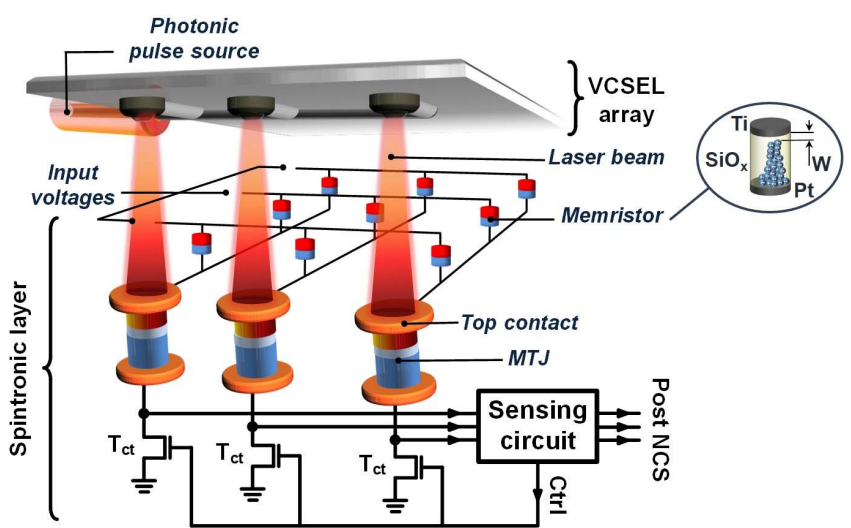

Fig. 2. The schematic view of the novel thermally assisted MTJ-based NCS. The MTJ and memristor act as neuron and synapse, respectively. 
significantly by increasing the temperature of the STNO using a nanosecond laser pulse. In fact, increasing the temperature of the STNO will decrease its energy barrier, which leads to a low energy and low delay STNO oscillation. As a result, the energy consumption and the delay of the LAO-NCS decrease compared with typical spintronic-based NCS.

The neuron consists of the STNO and an activating transistor in series. Considering the fact that the transistor acts as a switch, heating it up has no significant impact on the overall performance of the LAO-NCS. The on-chip laser spot size in nanometer regime can be achieved through Vertical Cavity Surface Emitting Laser (VCSEL) [29-31]. The output power of VCSEL can be tuned through changing the supply voltage of its driver [31]. Hence, a CMOS interfacing circuit can easily control the laser output power by manipulating the supply voltage of the laser diode driver.

\section{RESULTS}

In order to evaluate the LAO-NCS, first, the effect of elevating the temperature on the STNO characteristics is measured. Then, based on the measured results, a behavioral model of STNO is designed to be used in HSPICE. The simulations of the LAO-NCS are performed by HSPICE simulator in $65 \mathrm{~nm}$ CMOS technology. For memristor, the threshold adaptive memristor (TEAM) model [32] benchmarked with [25] is used.

\section{A. Experimental measurement}

In order to explore the effect of rising temperature on STNO characteristics, we used the STNO stack structure of Substrate/(100) $\mathrm{Al}_{2} \mathrm{O}_{3} /(3) \mathrm{Ta} /(30) \mathrm{CuN} /(5) \mathrm{Ta} /(17) \mathrm{Pt}_{38} \mathrm{Mn}_{62}$ /(2) $\mathrm{CoFe}_{30} /(0.85) \quad \mathrm{Ru} /(2.6) \quad \mathrm{CoFe}_{40} \mathrm{~B}_{20} / \mathrm{MgO}$ wedge/(1.4) $\mathrm{CoFe}_{40} \mathrm{~B}_{20} /(10) \mathrm{Ru} /(150) \mathrm{Cu} /(30) \mathrm{Ru}$ (thicknesses in nm). The $\mathrm{CoFeB}$ free layer has in-plane magnetization. The stack has the circular shape with diameter of $175 \mathrm{~nm}$. Fig. 3 (a) and (b) show the microscopic image of the STNO sample and the schematic view of deposited layer stack, respectively.

To evaluate the output power of STNO at different temperatures, the experimental setup of Fig. 3 (c) is utilized. The bias current is injected to the STNO through $T_{1}$ and $T_{2}$ terminals of the bias-tee. In case the bias current will be high enough, it leads to oscillation of STNO resistance. This resistance oscillation will provide a micro-volt oscillation at $\mathrm{T}_{3}$ terminal of bias-tee. Finally, the micro-volt oscillation of the STNO is amplified by an amplifier and will be injected to spectrum analyzer in order to measure the oscillation characteristics of STNO. The heating plate is used to set the temperature of STNO at different temperatures above room temperature. Fig 4 (a) shows the PSD measured at different temperature from $27^{\circ} \mathrm{C}$ to $100^{\circ} \mathrm{C}$ for $230 \mu \mathrm{A}$ bias current (the curves are offset by $10 \mu \mathrm{V}^{2}$ along the vertical axis for clarity). Noted, the impedance mismatch in the acquired spectrum must be considered. The input impedance of the amplifier is $50 \Omega$. Hence, considering the resistance mismatch between the amplifier and the STNO, the measured output power is only a fraction of actual emitted power of the STNO. In order to eliminate the effect of impedance mismatch, the integrated matched output power $\mathrm{P}_{\text {out }}$ of each device is calculated as follows [9]:

$$
P_{\text {out }}=P_{\text {measured }}\left(\frac{\left(R_{S T N O}+R_{A m p}\right)^{2}}{4 R_{S T N O} \cdot R_{A m p}}\right)
$$

where $\mathrm{R}_{\mathrm{STNO}}$ and $\mathrm{R}_{\mathrm{Amp}}$ are the resistance of STNO and input resistance of the amplifier, respectively. $P_{\text {measured }}$ is the measured output power based on the spectrum analyzer output. Fig. 4 (b) shows the measured STNO resistance in Pand AP-state at different temperatures from $27^{\circ} \mathrm{C}$ to $100^{\circ} \mathrm{C}$. The AP-state resistance is decreased with increasing the temperature and the P-state resistance is almost constant as predicted by equation (3) and as shown experimentaly before [19-21]. As a result, the TMR ratio decreases by increasing the temperature (Fig. 4 (c)) that shows the typical behavior of MTJs as a function of the bias current. The matched output power $\left(\mathrm{P}_{\text {out }}\right)$ of STNO versus bias current at different temperatures from $27^{\circ} \mathrm{C}$ to $100^{\circ} \mathrm{C}$ is shown in Fig. 4 (d). By applying sufficient positive bias current, the oscillation will start and by further increasing the bias current, the oscillation amplitude increases which leads to higher output power. Although the decrease in TMR with bias and temperature appears detrimental, the total power increases as the input power increase dominates this TMR degradation. This is typically the case up to a certain point were the TMR decrease dominates or the breakthrough voltage of the tunnel barrier is reached. Noted, applying a negative bias current will not cause oscillation but increases the noise power which leads to higher $\mathrm{P}_{\text {out }}$ In order to eliminate the effect of noise on $\mathrm{P}_{\text {out }}$, the output power of negative bias currents are deducted from the output power of positive bias currents as shown in Fig. 4 (e). Elavating the temperature increases $\mathrm{P}_{\text {out }}$ as shown in Fig. 4 (d) and (e). This effect is also seen in spin Hall nano-oscillators (SHNOs) [33]. As a result, the minimum bias current needed to detect the STNO oscillation of the fired neuron by sensing circuit decreases. This decreases the general energy consumption of the STNO-based LAO-NCS as will be discussed in the next section.

\section{B. Power consumption}

The total power consumption of the LAO-NCS includes the power consumption of the spintronic layer (memristors

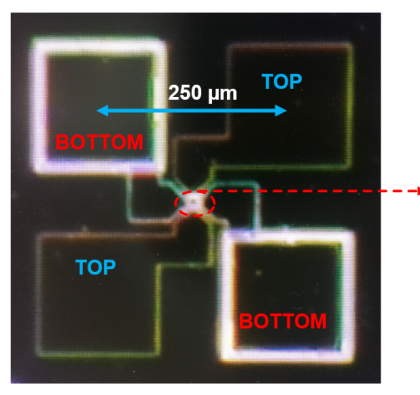

(a)

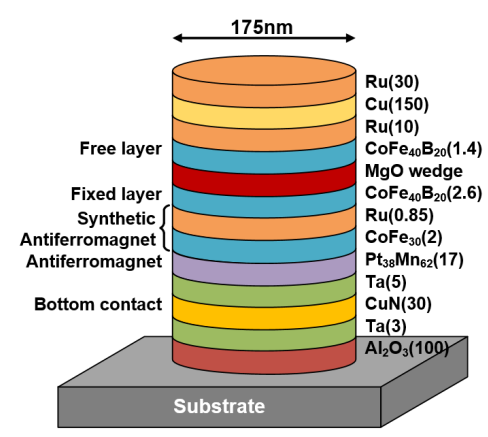

(b)

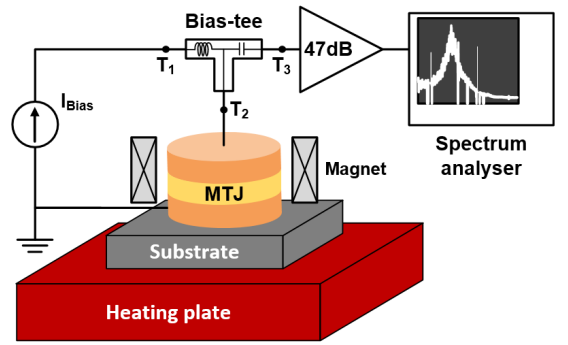

(c)

Fig. 3. (a) The microscopic image and (b) schematic view of the MTJ stack as STNO. (c) Schematic view of the experimental setup used for characterization of the STNO at different temperatures. 
and STNOs), the CMOS interfacing circuit and the laser. Due to the fact that the calibration phase is done just one time at the beginning of the NCS operation, its power consumption has no significant effect on the total power consumption of the LAO-NCS and can be ignored. Fig. 5 shows the power consumption of an STNO and the memristors connected the STNO to the inputs in spintronic layer at different temperatures. The power consumption of STNO is calculated by measuring the power consumption at the stimulation step (the minimum required power consumption leads to STNO oscillation with sensible output power). The power consumption of STNO decreases by $56 \%$ while increasing the temperature to $100^{\circ} \mathrm{C}$. This is due to the fact that heating up the STNO reduces its magnetization saturation and effective anisotropy field that tends to keep magnetization direction of FL aligned with the easy axis.

In order to calculate the power consumption of memristor array at elevated temperatures, first, the resistance reduction of memristors should be measured at different temperatures. However, the amount of resistance reduction not only depends on temperature, but also is a function of the initial resistance of memristor (weights) and the applied voltage (inputs). Hence, in order to estimate the average resistance reduction of memristor array, first, a 196x10 NCS is designed to recognize the handwritten digits in MATLAB. Then, using MNIST handwritten digits database [35] (size of the pictures is reduced to $14 \times 14$ ), the NCS is trained using 2000 training

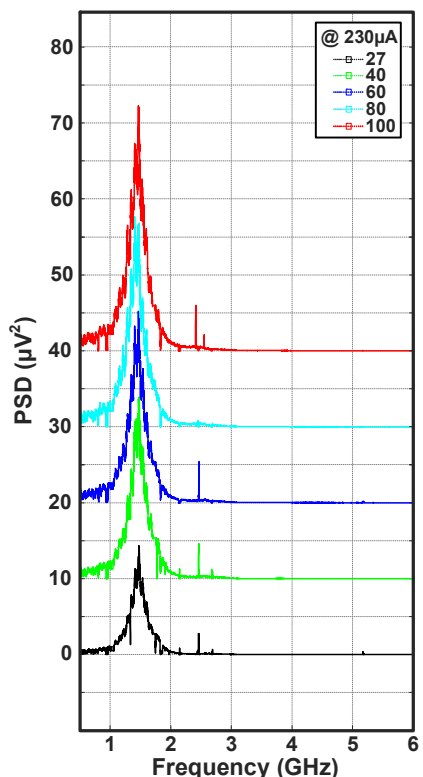

(a)

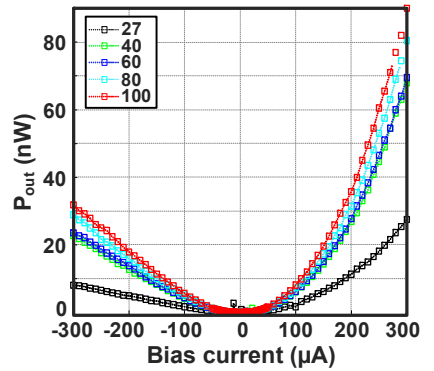

(d)

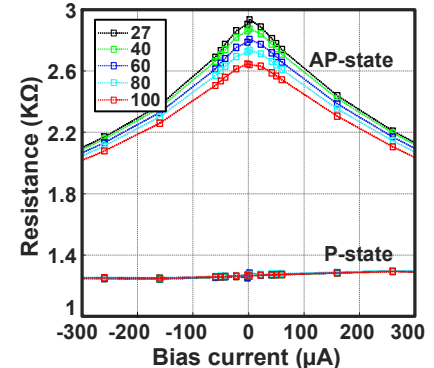

(b)

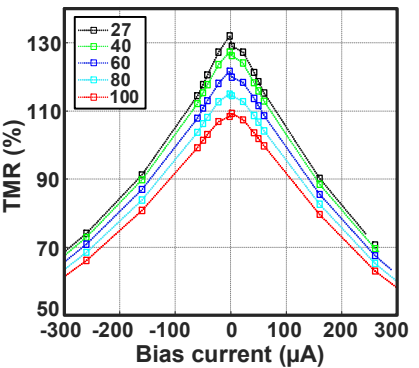

(c)

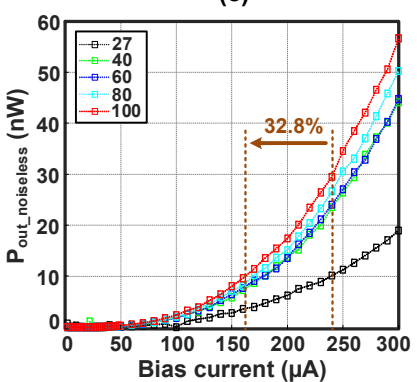

(e)
Fig. 4. (a) The PSD measured at different temperature from $27^{\circ} \mathrm{C}$ to $100^{\circ} \mathrm{C}$ for $230 \mu \mathrm{A}$ bias current, the curves are offset by $10 \mu \mathrm{V}^{2}$ along the vertical axis for clarity. (b) The AP and P states resistance, (c) the TMR ratio, (d) the integrated matched output power $\left(\mathrm{P}_{\text {out }}\right)$ and (e) the noiseless output power of STNO sample.

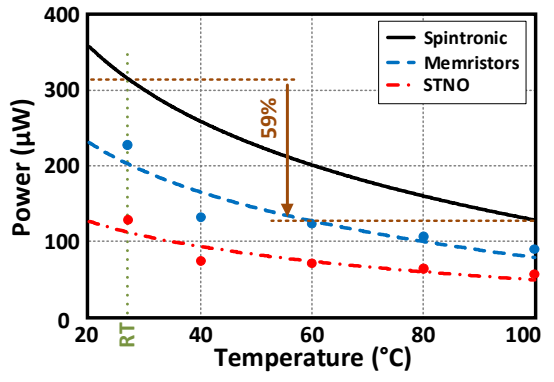

Fig. 5. The power consumption of an STNO and a column of memristors connecting the STNO to inputs at different temperature from room temperature to $100^{\circ} \mathrm{C}$.

images and the weights are extracted. Considering the facts that the negative weights cannot be implemented by memristors, the negative weights are considered as zero. In the next step, the weights are mapped to the resistance of memristors in the array. In order to model the effect of temperature increase on the memristor's resistance, an equation is fitted to the experimental results of [25] for each initial conductance from $0.2 \mu \mathrm{S}$ to $21 \mu \mathrm{S}$. Then, the fitted equations are used in MATLAB to determine the resistance of memristors at $100^{\circ} \mathrm{C}$. Finally, 10000 test images have been applied to the modeled NCS in MATLAB and the power consumption reduction of memristor array is calculated for each test image. The average power consumption reduction of a column of memristors connecting their corresponding STNO to inputs is $61 \%$ by increasing the temperature to $100^{\circ} \mathrm{C}$ (Fig. 5). This is due to the resistance reduction of memristors and lower bias current passing through them at elevated temperatures. This means that lower currents pass through smaller resistances in each stimulation phase. The power consumption of the CMOS interfacing circuit and the laser source are stimated to be $15 \mu \mathrm{W}$ and $25 \mu \mathrm{W}$ to keep the temperature of spintronic layer around $100^{\circ} \mathrm{C}$ [36-37]. Noted, scaling the STNO technology (smaller STNO volume) leads to lower laser power consumption. Considering the fact that the power consumption improvement in the spintronic layer is much higher than the power consumption of the laser and the CMOS interfacing circuit, the total power consumption of the LAO-NCS decreases by $46 \%$ at $100^{\circ} \mathrm{C}$ compared with room temperature.

\section{CONCLUSION}

To reduce the power consumption of future STNO-based NCSs, a microwatt-nanosecond laser pulse is utilized for the first time to ease the magnetic oscillation of the STNO through heating. The power consumption of the spintronic layer and the total power consumption of the proposed LAO-NCS are improved by $59 \%$ and $46 \%$ at $\mathrm{T}=100^{\circ} \mathrm{C}$ compared with operation at the room temperature. Noted, scaling the technology and increasing the temperature above $100^{\circ} \mathrm{C}$ leads to further improvement of the power consumption.

\section{REFERENCES}

[1] U.S. Department of energy, "Neuromorphic computing: from materials to systems architecture," Oct. 2015.

[2] IBM Research: Brain-inspired chip. [online] Available at: http://www.research.ibm.com/articles/brain-chip.shtml

[3] SpiNNaker. [online] Available at: http://apt.cs.manchester.ac.uk/ projects/SpiNNaker/

[4] Europe's Human Brain Project (HBP). [online] Available at: https://www.humanbrainproject.eu/ 
[5] DeepMind. [online] Available at: https://deepmind.com/

[6] X. Fong et. al. "Spin-transfer torque devices for logic and memory: prospects and perspectives," IEEE Trans. on Computer-Aided Design of Integrated Circuits and Systems (TCAD), vol. 35, no. 1, pp. 1-22, Jan. 2016.

[7] K. Yogendra, D. Fan, and K. Roy, "Coupled Spin Torque Nano Oscillators for Low Power Neural Computation," IEEE Trans. on Magnetics, vol. 51, no. 10, Oct. 2015.

[8] K. Yogendra et. al. "Magnetic Pattern Recognition Using InjectionLocked Spin-Torque Nano-Oscillators," IEEE Trans. On Electron Devices, vol. 63, no. 4, Apr. 2016.

[9] J. D. Costa et al., "High power and low critical current density spin transfer torque nano-oscilltors using $\mathrm{MgO}$ barriers with intermediate thickness," Nature Sci. Rep., vol. 7, pp. 7237, Aug. 2017.

[10] S. Fukami et al., "A spin-orbit torque switching scheme with collinear magnetic easy axis and current configuration," Nature Nanotechnology, vol. 11, pp. 621-625, Mar. 2016.

[11] G. Csaba and W. Porod, "Computational Study of Spin-Torque Oscillator Interactions for Non-Boolean Computing Applications," IEEE Trans. on Magnetics, vol. 49, no. 7, Jul. 2013.

[12] W.H. Rippard, M.R. Pufall, S. Kaka, T.J. Silva and S.E. Russek, "Injection Locking and Phase Control of Spin Transfer Nano oscillators", Physical Review Letters, PRL 95, 067203 (2005).

[13] W. Rippard, M. Pufall and A. Kos, 'Time required to injection-lock spin torque nanoscale oscillators", Applied Physics Letters 2013, Vol 103(18).

[14] A. Slavin et al., "Phase locking of a spin-troque nano-oscillator to a strong microwave magnetic field", APS meeting 2010, Vol 55, No.2.

[15] S. Kaka et al., "Mutual phase-locking of microwave spin torque nanooscillators," Nature Lett., vol. 437, pp. 389-392, Sep. 2005.

[16] X. Fong et al., Purdue Nanoelectronics Research Laboratory MTJ Model. nanoHUB. (2014), doi:10.4231/D33R0PV04.

[17] N. W. Ashcroft and N. D. Mermin, Solid states physics, Saunders college, 1976.

[18] J. G. Alzate et al., "Temperature dependence of the voltage-controlled perpendicular anistropy in nanoscale $\mathrm{MgO}|\mathrm{CoFeB}| \mathrm{Ta}$ magnetic tunnel junctions," Applied physics letters, vol. 104, no. 11, pp. 112410, Mar. 2014.

[19] J. M. Teixeira et al., "Evidence of spin-polarized direct elastic tunneling and onset of superparamagnetism in $\mathrm{MgO}$ magnetic tunnel junctions," Physical review B, vol. 81, no. 13, pp. 134423, Apr. 2010.

[20] Y. Takeuchi et al., "Temperature dependence of energy barrier in CoFeB-MgO magnetic tunnel junctions with perpendicular easy axis," Applied Physics Letters, vol. 107, no. 15, pp. 152405, Oct. 2015.

[21] B. Hu et al., "Temperature dependence of spin-dependent tunneling conductance of magnetic tunnel junctions with half-metallic Co2MnSi electrodes," Phys. Rev. B, vol. 94, pp. 094428, Sep. 2016.

[22] W. F. Brown, "Thermal fluctuations of a single-domain particle," Physical review, vol. 130, no. 5, pp. 1677, Jun. 1963.
[23] J. C. Sankey et al., "Mechanisms limiting the coherence time of spontaneous magnetic oscillations driven by dc spin-polarized currents," Physical review B, vol. 72, no. 22, pp. 224427, Dec. 2005.

[24] K. Yogendra et al., "Coupled spin-torque nano-oscillator-based computation: a simulation study," ACM journal on emerging technologies in computing systems, vol. 13, no. 4, pp. 56, Jul. 2017.

[25] C. E. Graves et al., "Temperature and field-dependent transport measurements in continuously tunable tantalum oxide memristors expose the dominant state variable," Applied Physics Letters, vol. 110, no. 12 , pp. 123501 , Mar. 2017.

[26] J. Torrejon et al., "Neuromorphic computing with nanoscale spintronic oscillators," Nature Letters, vol. 547, pp. 428-431, Jul. 2017.

[27] H. Farkhani et al., "A low-power high-speed spintronics-based neuromorphic computing system using real time tracking method," IEEE Journal on emerging and selected topics in circuit and systems (JETCAS), vol. 8, no. 3, pp. 627, Mar. 2018.

[28] H. Farkhani et al., "An energy efficient neuromorphic computing system using real time sensing method," IEEE Biomedical Circuits and Systems Conference (BioCAS), 2017.

[29] Z. Zhou, B. Yin and J. Michel, "On-chip light sources for silicon photonics," Nature Light: Science \& Applications, vol. 4, e358, Aug. 2015.

[30] C. Chen et al., "Sharing and Placement of On-chip Laser Sources in Silicon-Photonic NoCs," IEEE/ACM International Symposium on NoCS, pp. 88-95, 2014.

[31] V. Kozlov and A. C. Carusone, "Capacitively-Coupled CMOS VCSEL Driver Circuits," IEEE Journal of Solid-State Circuits, vol. 51, no. 9, pp. 2077, Sep. 2016.

[32] S. Kvatinsky et al., "TEAM: threshold adaptive memristor model," IEEE Trans. on Circuits and Systems-I: Regular Papers, vol. 60, no. 1, pp. 211, Jan. 2013.

[33] M. Tarequzzaman et al., "Spin torque nano-oscillator driven by combined spin injection from tunneling and spin Hall current," Nature Communications Physics, vol. 2, Feb. 2019.

[34] T. Maruyama and T. Morishita, "Copper nitride and tin nitride thin films for write-once optical recording media," Applied Physics Letters, vol. 69, pp. 890-891, Jun. 1998.

[35] Y. LeCun, L. Bottou, Y. Bengio, and P. Haffner, "Gradient-based learning applied to document recognition," Proc. IEEE, vol. 86, no. 11, pp. 2278-2324, Nov. 1998.

[36] H. Farkhani et al., "LAS-NCS: A Laser-Assisted Spintronic Neuromorphic Computing System," IEEE Trans. on Circuits and Systems II: Express Briefs, Early Access, DOI: 10.1109/TCSII.2019.2908077, 2019.

[37] T. Böhnert et al., "Magnetic tunnel junctions with integrated thermometers for magnetothermopower measurements," Journal of Physics: Condensed Matter, vol. 29, pp. 185303, Apr. 2017. 